\title{
Practical considerations on the restoration of sexual function in the hypogonadal man
}

\author{
Alvaro Morales MD FRCSC
}

A Morales. Practical considerations on the restoration of sexual function in the hypogonadal man. J Sex Reprod Med 2001;1(2):109-112.

Insidious hypogonadism that is associated with aging has most of the same manifestations as andropause, but its development is subtle. A response to treatment is usually prompt in hypogonadal men experiencing sexual dysfunction. It is recommended that patients with hypogonadism and sexual dysfunction receive treatment for three months. In the absence of a response at that time, comorbidities should be sought out actively. The results of treatment are not spectacular. This observation reflects the fact that, in aging men, hormonal shifts are only one of many other comorbid conditions (eg, atherosclerosis, peripheral neuropathy, emotional disturbances).

Key Words: Androgens; Intramuscular injections; Oral route; Percutaneous gel; Transdermal patch

\section{ANDROGEN DEFICIENCY IN THE AGING MALE AND SEX: CLINICAL PICTURE}

In contrast to menopause, the process of androgen deficiency in the aging male or andropause is, most commonly, characterized by an insidious onset and a very slow progression. The clinical picture can easily be attributed to the natural and unavoidable consequences of aging. True andropause is seen in the hormonal ablative treatment of advanced prostate cancer, and in this condition, the mani-

\section{Observations pratiques relatives au rétablissement du fonctionnement sexuel chez les hommes atteints d'hypogonadisme}

RÉSUMÉ : L'hypogonadisme insidieux associé au vieillissement présente à peu près les mêmes caractéristiques que l'andropause, mais celui-ci s'installe plus subtilement. La réaction au traitement est généralement rapide chez les hommes hypogonadiques présentant un dysfonctionnement sexuel. Il est recommandé de soumettre les hommes souffrant à la fois d'hypogonadisme et de dysfonctionnement sexuel à un traitement hormonal pendant trois mois. En l'absence de réaction, il faudrait chercher d'autres maladies concomitantes. Par ailleurs, les résultats du traitement ne sont pas spectaculaires. La remarque met en évidence le fait que le changement hormonal chez les hommes vieillissants ne constitue qu'une manifestation parmi tant d'autres, par exemple l'athérosclérose, la neuropathie périphérique, les troubles émotifs.

festations are defined more clearly. Soon after chemical or surgical castration, there is a rapid decline in sexual interest and erectile function. Although rare, some men with very low levels of testosterone after castration retain some libido and, even more rarely, sexual erections. Nocturnal erections universally disappear in patients with profound hypogonadism. The insidious hypogonadism associated with aging has most of the same manifestations as andropause, but its development is subtle (1). 
TABLE 1

Most frequently used testosterone preparations

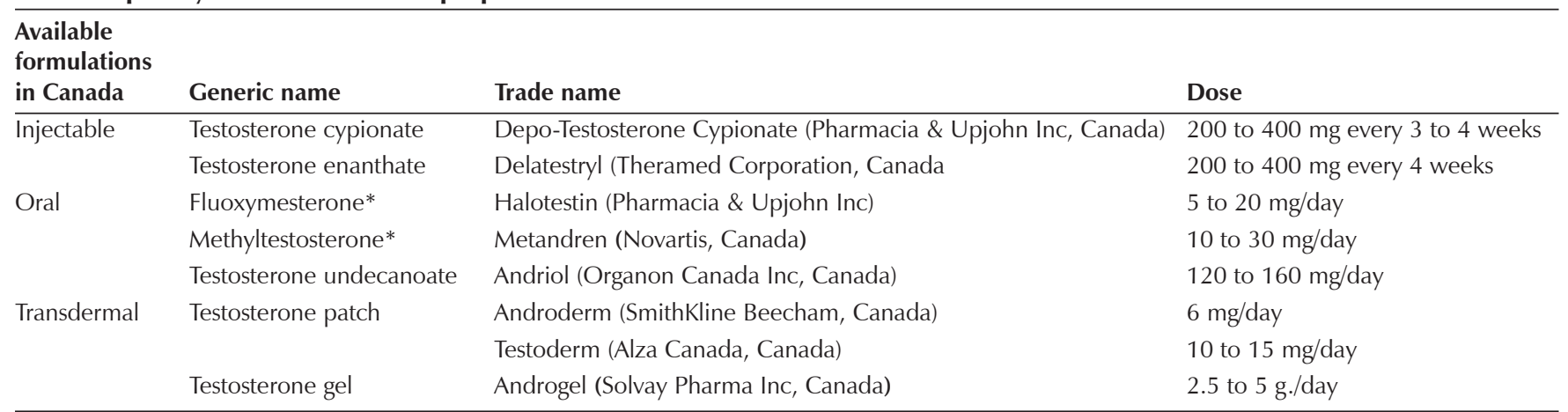

*Just as with 17-alpha alkylated testosterone products, both fluoxymesterone and methyltestosterone are associated with serious liver toxicity

The andropause syndrome is characterized by the prominent and easily recognizable features of diminished sexual desire and erectile quality, particularly nocturnal erections; changes in mood, with concomitant decreases in intellectual activity and spatial orientation, fatigue, depression and anger; decreased lean body mass, with associated diminution in muscle volume and strength; decreased body hair and skin alterations; decreased bone mineral density, resulting in osteoporosis; and increased visceral fat. These manifestations need not all be present to diagnose the syndrome. In addition, the severity of one or more of the above symptoms does not necessarily match the severity of the other symptoms, and the uneven presence of these manifestations is not yet understood. Sexual dysfunction, however, is recognized as one of the most constant features of andropause.

\section{THERAPEUTIC OPTIONS}

Therapy of hypogonadism is generally medical, as opposed to surgical (pituitary tumours), and effective. For the clinician, the goals of treatment most commonly include the restoration of sexual functioning, libido and a sense of well-being. As indicated in previous papers in the present issue, it is equally important that androgen replacement can prevent or improve already established osteoporosis, optimize bone density, restore muscle strength, improve mental acuity and normalize growth hormone levels, especially in elderly men. Testosterone replacement therapy should maintain not only physiological levels of serum testosterone, but also the metabolites of testosterone, including dihydrotestosterone (DHT) and estradiol $\left(\mathrm{E}_{2}\right)$ to optimize the maintenance of libido, virilization and sexual function. It is worth noting that in the Massachusetts Male Aging Study, the only sex hormone correlating with sexual dysfunction was dehydroepiandrosterone. This sole correlation, of course, points to the shortcomings of epidemiological data available about a condition as complex as erectile dysfunction.

Current treatment options of hypogonadism include oral tablets or capsules; intramuscular (IM) preparations (longand short-acting); implantable, long-acting, slow-release pellets; and transdermal patches (scrotal and nonscrotal). Neither injectable preparations nor slow release pellets reproduce the circadian pattern of testosterone production of the testes. The latter is best accomplished by dermal patches; however, oral testosterone may also approximate a circadian rhythm by dose adjustments. Common testosterone preparations are shown in Table 1.

\section{Oral preparation}

Oral preparations of testosterone require special consideration. Oral agents that are available include alkylated androgen preparations (to prevent rapid hepatic metabolism), which generally result in erratic androgenic effects and significant changes in lipid profile, and have a high risk of adverse liver side effects (2). The hepatotoxicity causes hepatocellular adenoma, cholestatic jaundice and hemorrhagic liver cysts (3). In addition, alkylated androgens may result in increased low density lipoprotein and decreased high density lipoprotein levels, with the resultant potential for increased cardiovascular risks (4). Testosterone undecanoate is widely used throughout the world (with the notable exception of the United States). As a testosterone ester, it is free of liver toxicity and effective in bringing levels of serum testosterone to within physiological range. It may, however, result in supraphysiological levels of DHT and rare gastrointestinal side effects (5). The drug is lipid soluble and should be taken with meals. The recommended dose is 120 to $200 \mathrm{mg}$ in three divided doses, depending on the degree of testosterone deficiency, body surface, obesity and clinical response.

\section{IM injections}

IM injections of testosterone are usually long-acting. These formulations obtain a maximum concentration approximately $72 \mathrm{~h}$ after injection, which slowly diminishes over the ensuing 10 to 14 days. The gradual decline frequently results in a very low nadir before injection is repeated (6). Parenteral androgens do not provide normal circadian patterns of serum testosterone, and the injections are somewhat uncomfortable. Levels of DHT are normal, but androgen 
metabolites are frequently higher than the expected normal range, while $\mathrm{E}_{2}$ levels may become excessive in some men.

The most widely used IM preparations include the 17-beta-hydroxyl esters of testosterone, which are administered with slow-release, oil-based injection vehicles. These esters, however, lack inherent androgenic activity and must be hydrolyzed to testosterone before they become pharmacologically active. The 17-beta-hydroxyl esters of testosterone include the shorter-acting testosterone proprionate, and the longer-acting testosterone enanthate and cypionate. Testosterone propionate is rarely used clinically because of its short half-life and the requirement for injection every other day to maintain normal serum testosterone levels. Testosterone enanthate and cypionate, however, may be administered every 10 to 21 days to maintain average testosterone levels. It must be acknowledged, however, that testosterone levels with these preparations surge to supraphysiological levels. The decline in serum testosterone levels continues over 14 to 21 days, reaching baseline at approximately 21 days.

The significant peaks and valleys (roller coaster effect) of serum testosterone levels in patients treated with parenteral testosterone injections may produce significant mood swings, and noticeable increase and decreases in libido and sexual function. These long-acting testosterone preparations are, however, the most cost efficient methods of androgen replacement. The dose is 200 to $400 \mathrm{mg}$ every two to four weeks. Injections of $200 \mathrm{mg}$ maintain normal testosterone levels for approximately two weeks, while $300 \mathrm{mg}$ doses are required to maintain eugonadal range for approximately three weeks. Higher doses do not maintain testosterone levels in the normal range beyond the three-week limit. These testosterone preparations have clearly been shown to improve libido, sexual function, potency, energy level, bone density and mood, if these abnormalities are caused by androgen deficiency. Supraphysiological levels of serum testosterone may result in infertility due to the suppression of follicle-stimulating hormone and luteinizing hormone production (7). Reports of sexual aggressiveness and overall aggressive behaviour during peak levels achieved after injectable testosterone treatment are anecdotal. Careful counselling about the possibility of these mood and behavioral changes for patients undergoing parenteral testosterone therapy is essential.

Transdermal testosterone therapy, a more expensive but more physiological approach to testosterone replacement, recently became available worldwide (8). Transdermal testosterone therapy is available in both scrotal and nonscrotal patches. These preparations use elemental testosterone that is absorbed transdermally to obtain normal serum testosterone levels and reproduce the diurnal physiological variations of testosterone observed in normal human testosterone secretion. Patches are applied at bedtime, with peak testosterone levels achieved in the early morning and a nadir seen before patch replacement. Transdermal patches are now available for nonscrotal use, and include the Androderm (Smithkline Beecham, Canada) and Testoderm
(Alza Canada, Canada) systems $(9,10)$. These nonscrotal patches also maintain diurnal serum concentrations, with normal $E_{2}$ and DHT levels. While most patients can be treated with a single nonscrotal transdermal patch, patients need varying amounts of testosterone replacement, depending on the patients' deficiency and absorption characteristics. Morning testosterone levels should be evaluated within two to three weeks of initiating therapy to identify peak testosterone levels. Serum evaluations of these patients should be carried out between 08:00 and 10:00 to determine the highest daily level of serum testosterone produced by the patch. Because androgen levels with these systems do not increase beyond normal, it is unlikely that detrimental changes in mood occur with transdermal preparations. While psychological effects have not been carefully studied in a long term series, no cases of aggressiveness with the transdermal patch systems have been reported (11). Follow-up studies of transdermal testosterone replacement have, however, showed an improvement in testosterone levels associated with improved sexual function, libido and nocturnal penile tumescence response, with the maintenance of normal hematocrit levels, lipid profile, prostate-specific androgen (PSA) levels and prostatic volumes (12). The most common negative effects associated with the dermal patches include the inconvenience of applying them and dermatitis, which sometimes leads to significant chemical burns (13).

A transdermal testosterone hydroalcoholical gel preparation (Androgel, Solvay Pharma Inc, Canada ) is a new addition to the armamentarium. When applied to skin, it dries up in less than $5 \mathrm{~min}$, and early reports indicate that it is well tolerated (insignificant skin irritation) and can be applied to the same site without increasing dermatological problems or decreasing efficacy. Regarding efficacy, administration of the testosterone gel results in the normalization of the serum values of testosterone. The levels of DHT tend to be above normal. The experience with this delivery form is short, and there is a lack of dose-ranging studies (14). Nevertheless, short term studies (six months) indicate that this preparation results in improved sexual function and mood, and increased lean body mass and muscle strength that are associated with a decrease in fat mass (15). These studies are the most compelling to date, but are very limited in sample size and the length of follow-up.

\section{RESULTS OF TREATMENT}

The benefit of dealing with hypogonadal men experiencing sexual dysfunction is that a response to treatment is usually prompt. It is recommended that patients with hypogonadism and sexual dysfunction receive treatment for three months. In the absence of a response at that point, comorbidities should be sought out actively. The results of treatment are not spectacular. This observation reflects the fact that, in aging men, hormonal shifts are only one of many other conditions (eg, atherosclerosis, peripheral neuropathy, emotional disturbances) that affect these patients. 


\section{REFERENCES}

1. Gooren LI. The age-related decline of androgen levels in men: clinically significant? Br J Urol 1996;78:763-8.

2. Morales A, Johnston B, Heaton JW, Clark A. Oral androgens in the treatment of hypogonadal impotent men. J Urol 1994;152:1115-8.

3. Bagatell CJ, Bremner WJ. Androgens in men - uses and abuses. N Engl J Med 1996;14;334:707-14.

4. Bagatell CJ, Bremner WJ. Androgen and testosterone effects on plasma lipids. Prog Cardiovasc Dis 1995:38:255-71.

5. Gooren LJG. A ten-year safety study of the oral androgen testosterone undecanoate. J Androl 1994;15:212-5.

6. Bhasin S. Clinical review 34: Androgen treatment of hypogonadal men. J Clin Endocrinol Metab 1992;74:1221-5.

7. Bhasin S, Bremner WJ. Emerging issues in androgen replacement therapy. J Clin Endocrinol Metab 1997;82:3-8.

8. McClellan KJ, Goa KL. Transdermal testosterone. Drugs 1998;55:253-8

9. Arver S, Dobs AS, Meikle AW, et al. Long-term efficacy and safety of a permeation-enhanced testosterone transdermal system in hypogonadal men. Clin Endocrinol 1999;47:727-37.

10. Cunningham GR, Cordero E, Thornby J. Testosterone replacement with transdermal therapeutical systems: physiologic serum testosterone and elevated dihydro testosterone levels. JAMA 1989;261:2525-30.

11. Findlay JC, Place V, Snyder PJ. Treatment of primary hypogonadism in men by the transdermal administration of testosterone. J Clin Endocrinol Metab 1989;68:369-73.

12. Meikle AW, Arver S, Dobs AS, et al. Effects of a permeation enhanced transtestosterone transdermal system on prostate parameters in previously treated or untreated hypogonadal males. Br J Urol 1996;77:38. (Abst)

13. Jordan WP, Atkinson LE. Comparison of the skin irritation potential of two testosterone transdermal systems: an investigational system and a marketed product. Clin Ther 1998;20:80-7.

14. Wang C, Berman N, Longstreth JA, et al. Pharmacokinetics of transdermal testosterone gel in hypogonadal men: application of gel at one site versus four sites: a General Clinical Research Center Study. J Clin Endocrinol Metab 2000;85:964-9.

15. Wang C, Swerdloff RS, Iranmanesh A, et al. Transdermal testosterone gel improves sexual function, mood, muscle strength and body composition parameters in hypogonadal men. J Clin Endocrinol Metab 2000;85:2389-853. 